\title{
1 Application of neural network to detect freezing of gait in patients with Parkinson's disease
}

\begin{abstract}
Freezing of gait (FOG) consistently reoccurs in the later phases of a patient suffering from Parkinson's disease (PD). Although it is treated with pharmacological treatment, the impact of the medication fades with increasing duration of the disease and thus diminishing the mobility of a patient. This chapter aims at developing a neural network-based classification model that helps to detect FOG episodes in a patient at early stages so that lethal mishaps can be avoided. In this application example, we build user-independent FOG recognition system that would work along in conjunction with nonpharmacological medications. The structured system of developing a neural network-based classification model can be organized into three different stages. The process starts with extraction of suitable features from the dataset. In the subsequent stage, patients are additionally grouped into two clusters depending on the FOG episodes. In the final stage, two neural network models are developed using feedforward network on the two clusters that were formed. The accuracy of the model is computed using sensitivity and specificity.
\end{abstract}

Keywords: healthcare, Parkinson's disease, freezing of gait, feature extraction, clustering, neural network

\subsection{Introduction}

Parkinson's disease (PD), caused by Parkinsonism, was first discovered by James Parkinson in 1817. This symptom is observed due to a decrease in the neuromelanin neuron called dopamine causing unusual activity in the cerebrum [1]. Main features of PD are frequently categorized into four groups: trembling, rigidity, akinesia, and posture imbalance [2]. The Hoehn-Yahr score (H\&Y) is used to measure different stages of $\mathrm{PD}$ in a patient, which are classified into five distinct stages indicating relative disability levels as follows [3, 4]:

Stage 1: Affects only one side of the body with minimal or no functional impairment.

Stage 2: Affects both sides of the body without the loss of balance.

Bharatendra Rai, Amruta Meshram, University of Massachusetts, Dartmouth, USA 
Stage 3: Impairment is found; functionality is restricted. In this stage, the patient is physically capable of doing work independently, and the disability is mild or moderate.

Stage 4: A higher level of disability, but the patient can still walk or stand without being assisted with a conspicuous concentration difficulty.

Stage 5: Bounded to a bed or a wheelchair, but needs an assistant.

Freezing of gait (FOG) is the short, inconstant loss or an explicit deterioration in the movement of the feet, despite inclination or willingness to walk $[5,6]$. Some common symptoms of a FOG episode involve difficulty in executing efficient stepping even when the patient makes an effort to do so, a feeling of difficulty to move legs, and also shivering of legs [6]. About $70 \%$ of FOG is observed in a patient during advanced stages of PD, but $26 \%$ of FOG is reported in the beginning stages for those patients who have not undergone a levodopa therapy yet [5]. FOG experienced by a patient is mostly a brief episode that can sometimes last more than $1 \mathrm{~min}$ [7].

As the mechanism of the freezing experienced by a patient is multifactorial and not well understood, the treatment to find a solution is very challenging [8]. Levodopa is the most efficient drug used for PD [9]. Some other drugs that are available are dopamine agonists, catechol-o-methyl-transferase inhibitors, and nondopaminergic agents, but the effect of these drugs does not stay for a longer duration in patients [9].

Although the complete cure of PD is not available at this time, if FOG events can be predicted even a few seconds before they occur for a patient, nonpharmaceutical treatments can be very helpful to avoid fatal accidents. These nonpharmaceutical treatments may take the form of visual, auditory, and tactile cueing provided to the patient [10].

The objective of this chapter is to build a user-independent classification model by application of neural network techniques to enhance FOG identification in PD. To accomplish this objective, the data related to patients are partitioned into two clusters depending on the features and then we develop a feedforward neural network model for each of the two groups.

\subsection{Data and approach}

In this chapter, we will use Daphnet FOG dataset which is freely available [4]. This dataset is based on experiments that were performed at the Laboratory for Gait and Neurodynamics at the Department of Neurology of the Tel Aviv Sourasky Medical Centers (TASMC). The dataset consists of FOG events captured with the help of wearable accelerometer sensors.

Figure 1.1 provides a representation of the sensor data that will be used in this chapter. In this study, three sensors are utilized to quantify acceleration in three 


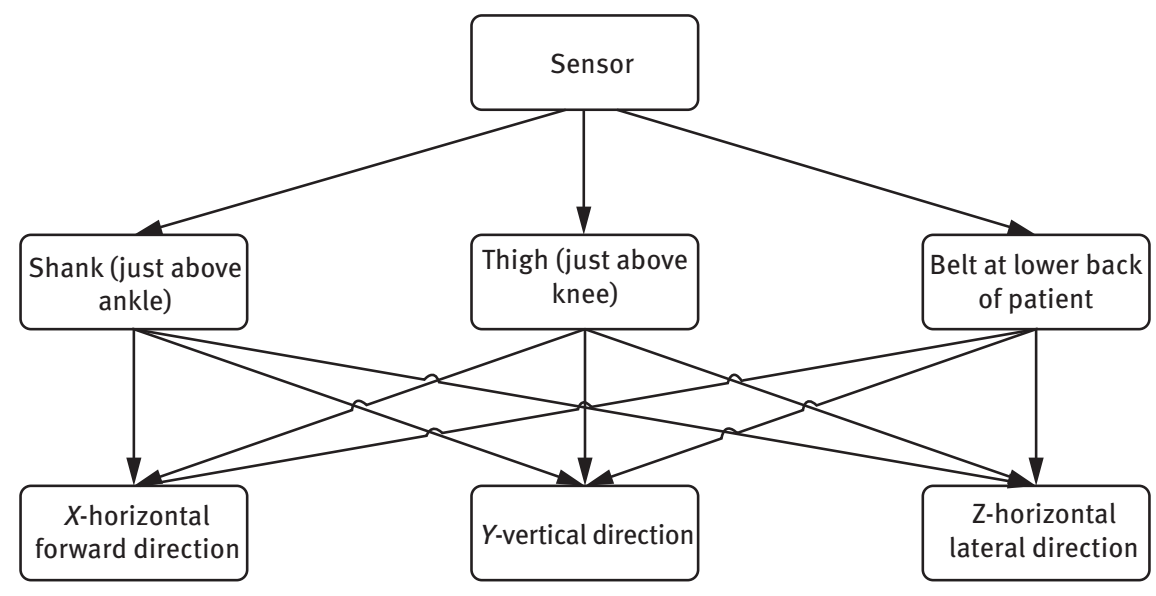

Figure 1.1: Sensor usage flowchart.

dimensions: first appended at the shank, second at the thigh, and third at the lower back. Every sensor collects reading from movements related to three axes: $X$-axis, $Y$-axis, and $Z$-axis. The horizontal forward direction reading is collected using $X$-axis, the vertical direction reading is collected using $Y$-axis, and the horizontal lateral information is collected using $Z$-axis. The dependent variable describes the presence or absence of the FOG. In total, the database consists of ten variables: one dependent and nine independent variables.

Figure 1.2 provides a flowchart depicting the data collection phase. From the flowchart we can observe that in all there are ten patients: three female patients and seven male patients. These ten patients have the disease span varying from 2 to 30 years. The age for ten patients ranges from 59 to 75 years. The patients are further classified as belonging to the "ON" phase of medication and "OFF" phase of medication. At the time of collecting data, each patient completes diverse walking patterns that involve an arbitrary walk including $360^{\circ}$ turn, walking in straight line including $180^{\circ}$ turn and walking while performing simulation tasks. All these events were recorded using a digital video camera. A physiotherapist is used for noting events during the experiments to determine start time, duration, and end time for the FOG event. In addition, the current activity of the patients is also recorded.

The data collection period involved recording for $8 \mathrm{~h}$ and $20 \mathrm{~min}$ with an interval of 15-20 ms. Out of the ten patients, eight patients experienced FOG episodes. Overall, 237 FOG episodes were detected during the experiment. Patient 5 encountered as many as 66 FOG episodes, whereas patient 6 experienced 10 FOG episodes which were least among all the patients with FOG. Figure 1.3 represents a boxplot of the duration of FOG events for each patient. Data of patient 4 and patient 10 are excluded from the plot as they did not experience any FOG event. Although patient 6 (disease duration: 22 years) experienced only 10 FOG events, we can observe from 


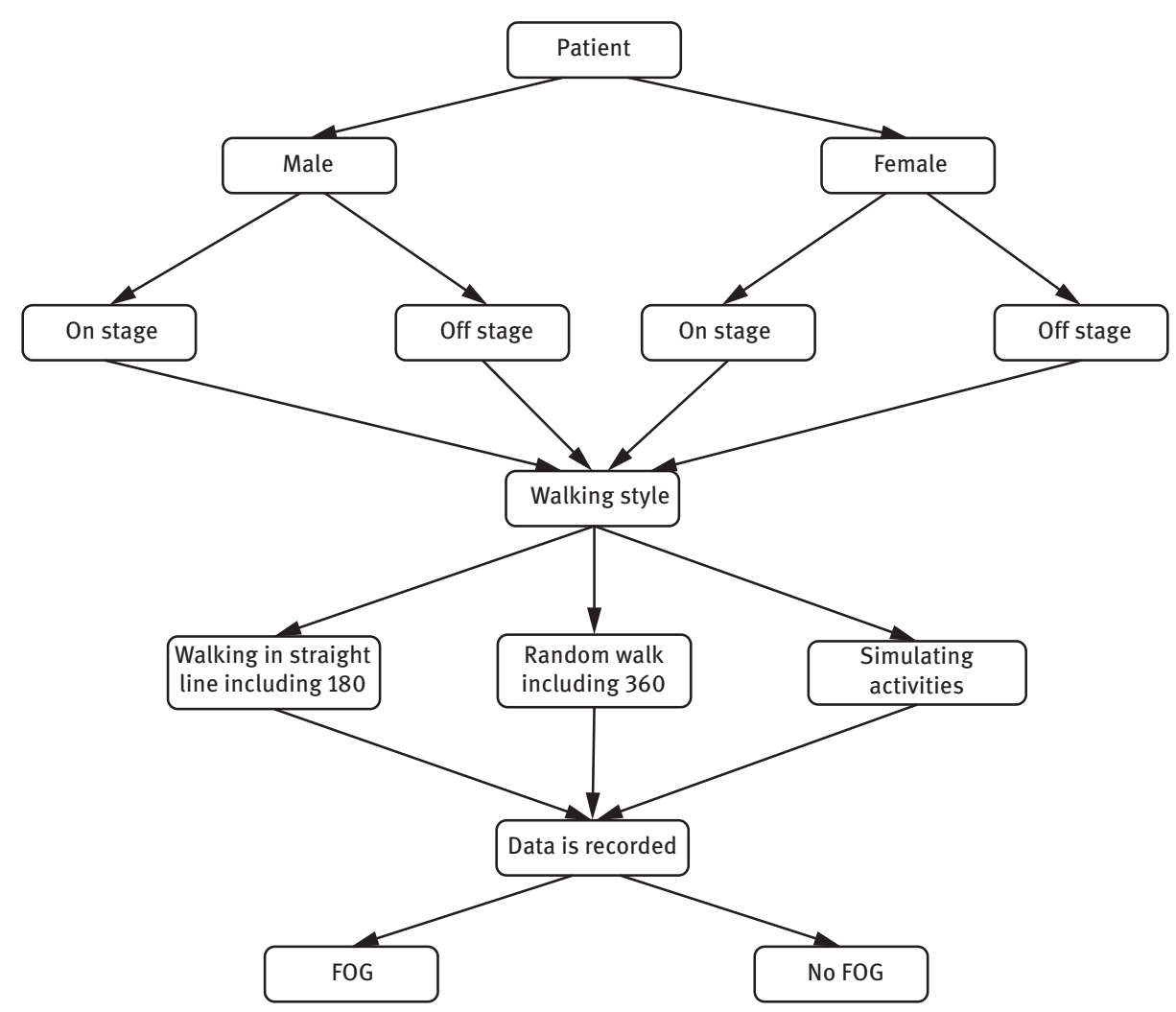

Figure 1.2: The patient data collection process.

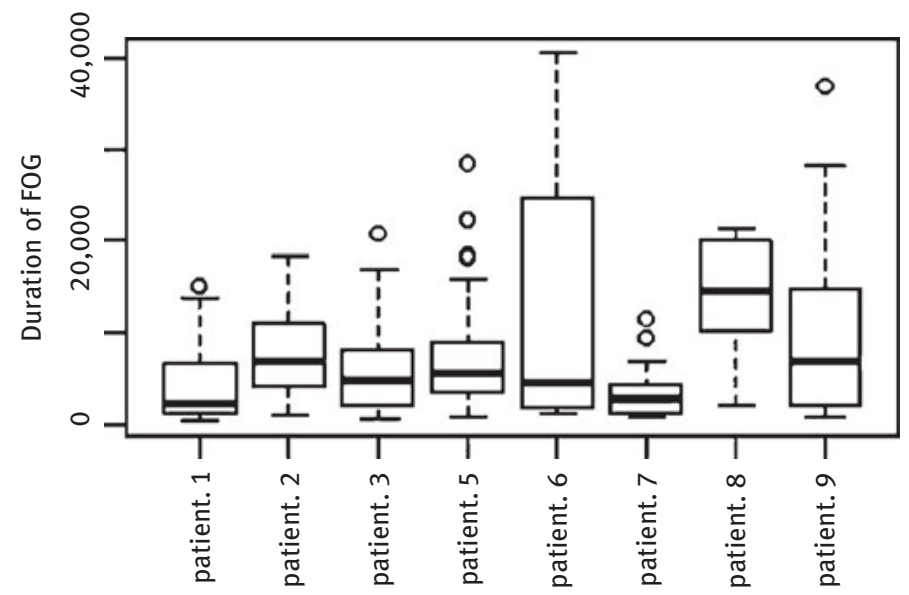

Figure 1.3: Boxplot for duration of FOG events in each patient (Patients 4 and 10 are excluded as they did not experience FOG events). 
the boxplot that a period of FOG events was the highest variability ( $\mathrm{sd}=14.32 \mathrm{~s}$ ) for this patient. Patient 8 (disease duration: 18 years) also indicates relatively high variability in length of FOG events.

On the other hand, patient-7 (disease duration two years) experienced the least variability ( $\mathrm{sd}=2.73 \mathrm{~s}$ ) among all patients. Patient- 8 was also observed to have the highest average FOG event duration (mean $=14.34 \mathrm{~s}$ ), and patient 7 had the least average (mean $=3.38 \mathrm{~s}$ ). It is to be noted that patient 8 was on the "ON" stage and was the only patient with the highest H\&Y score of 4.

\subsection{Methodology}

This section provides a detailed overview of the three stages of developing a classification model using neural network.

Stage 1: (Extracting essential feature for model building): In this analysis, use of a $50 \%$ overlapping window is utilized to derive 11 statistical features. The statistical features are derived from the nine variables and consist of mean: mean 1 (lower $10 \%$ of the values were trimmed), mean 2 (lower $20 \%$ of the values were trimmed), minimum, maximum, median, standard deviation, kurtosis, variance, skewness, and mode. Thus, in all we develop 99 features from the data.

Stage 2: (Clustering analysis for model building): In this phase, data with FOG episodes are considered for the analysis and the patients are grouped into clusters depending on their FOG episodes. FOG events using all 99 features were utilized to perform $k$-means cluster analysis. This analysis resulted in the formation of two distinct clusters: patients 1, 2, 3, and 5 are classified as cluster 1 , while patients $6,7,8$, and 9 are grouped as cluster 2 .

Stage 3: (Model building): In this stage, we build a neural network model to classify and predict FOG events. In this dataset, the overall percentage of FOG events is $9.7 \%$, which means the percentage of no-FOG event is $90.28 \%$. If we consider the worst scenario where without developing any classification model we make a prediction that all events belong to no-FOG category, then we will be right $90.28 \%$ of the time. We can use this number as a benchmark and develop a classification model that has a better performance. In this study, we will make use of sensitivity and specificity to assess the performance of the classification model. Sensitivity is defined as the ratio of FOG episodes that are correctly classified out of all the FOG episodes observed in the data. Whereas specificity is defined as the ratio of no-FOG episodes that are correctly classified out of all the no-FOG episodes observed in the data. 
To perform classification of the two clusters, feedforward networks (nntraintool) in MATLAB are utilized to classify inputs according to the target classes. While developing a classification model on the two clusters formed, $70 \%$ of the data are used as training data, $15 \%$ of the data are used as validation data, and the remaining $15 \%$ of the data are used as test data. The representation of crossentropy versus performance using training, validation, and test data for cluster 1 is depicted in Figure 1.4.

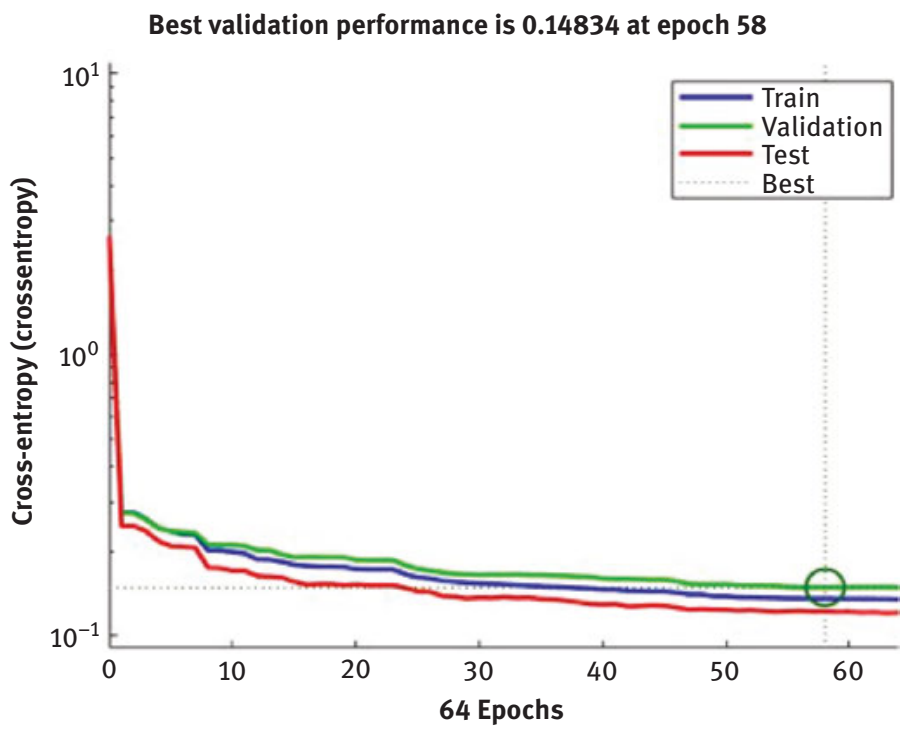

Figure 1.4: Cross-entropy versus performance using training, validation, and test data for cluster 1.

For developing a good classification model we minimize cross-entropy. As shown in the plot, the cross-entropy reduces with the increasing epochs for training, validation, and test data. The dotted green line indicates the best epoch, which in this case is 58 epochs. The performance of the network is measured using four lines that capture train, validation, test, and best situations. If any of the three lines (training, validation, and testing) meet or pass near the best (dotted) line, it symbolizes convergence.

To assess the classification performance of the mode, we develop a confusion matrix. Figure 1.5 provides the confusion matrix for cluster 1.

From the confusion matrix, we can observe that there is consistency in results for training, validation, and test data. For training data, the model correctly classified 8,855 events as no-FOG and 457 events as FOG. The sensitivity and specificity values for the training data are $66.4 \%$ and $96.1 \%$, respectively. The sensitivity and specificity values for the validation data are $63.1 \%$ and $96.3 \%$, respectively. Similarly, sensitivity and specificity values for test data are $64.4 \%$ and $97 \%$, respectively. This consistency 

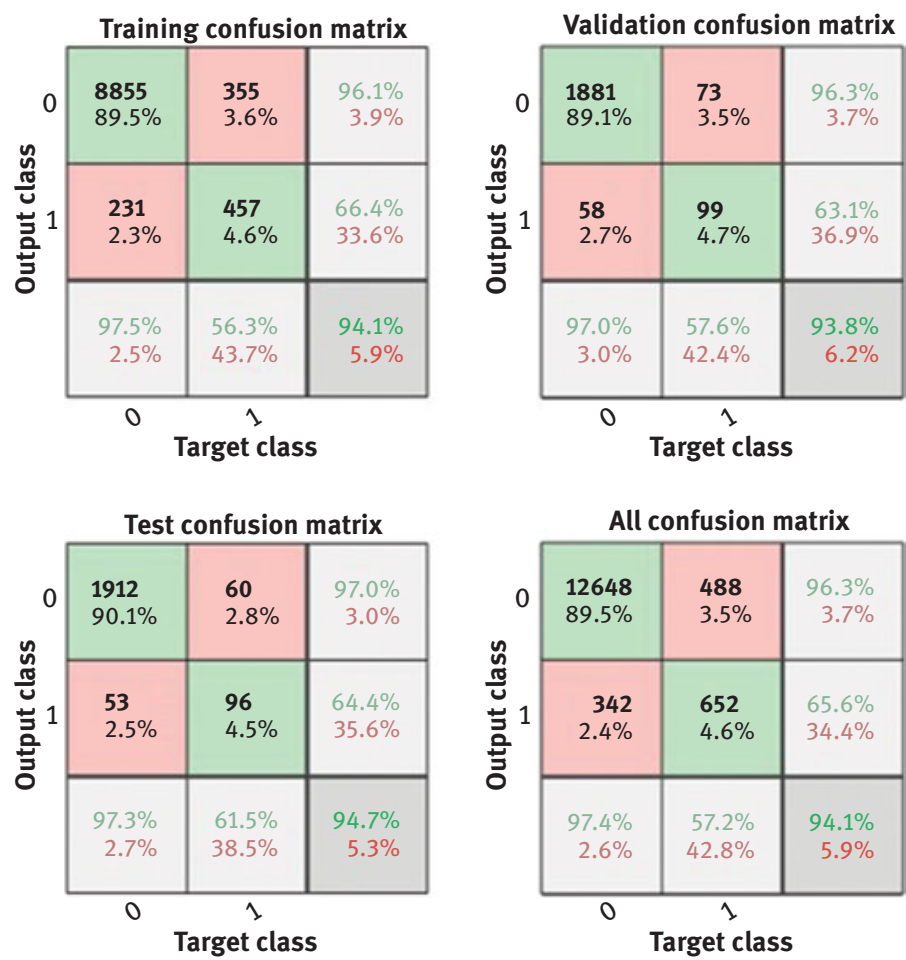

Figure 1.5: The confusion matrix for cluster 1.

in results across training, validation, and test data indicates the absence of overfitting problem. Another observation that we can make from this confusion matrix is about the events that are misclassified. It is observed that the events that belong to FOG category are misclassified as no-FOG at a slightly higher rate compared to a situation where events belonging to no-FOG category are misclassified as FOG. This pattern occurs consistently for the training, validation, and also the test data.

Figure 1.6 represents the receiver operating characteristics (ROC) curve of the feedforward network at epoch 58 for cluster 1.

From the ROC we observe that the classification performance for the training, validation, and test data are again consistently high. The diagonal line in the plot depicts the classification performance with 50\% accuracy. And closer the ROC curve to the upper left corner of the plot, better the classification performance.

The representation of cross-entropy versus performance using training, validation, and test data for cluster 2 is depicted in Figure 1.7.

As observed, the cross-entropy reduces with the increasing epochs in training, validation, and test data. The dotted green line indicates the best epoch, which is 75 epochs. 

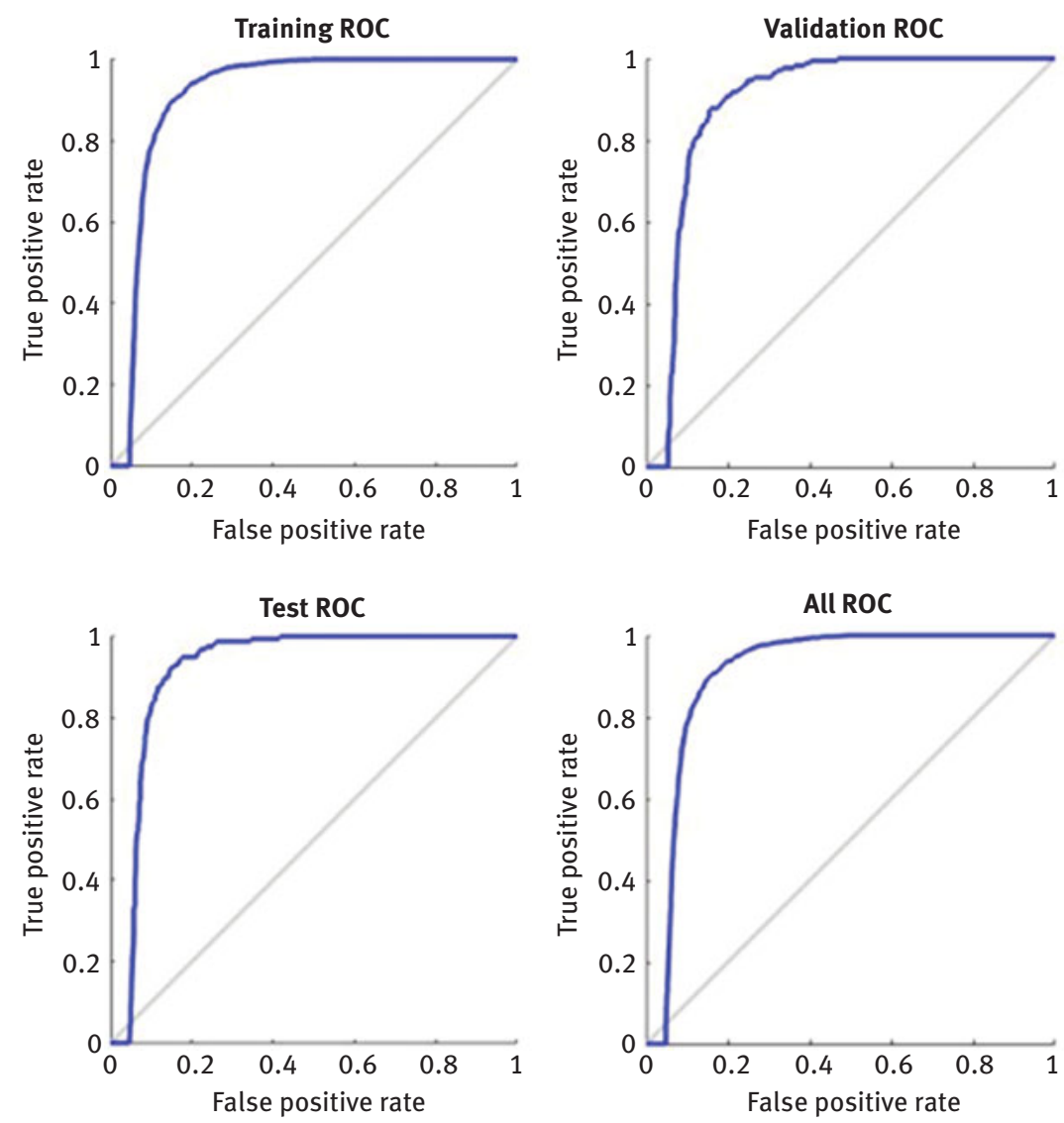

Figure 1.6: The ROC curve for a feedforward network at 58 splits for cluster 1.

Figure 1.8 provides the confusion matrix for classification model with cluster 2 .

From the confusion matrix we can observe that the sensitivity and specificity values for the training data are $63.7 \%$ and $91 \%$, respectively. The sensitivity and specificity values for the validation data are $71.6 \%$ and $91.9 \%$, respectively. Similarly, we observe that the sensitivity and specificity for test data are $74.6 \%$ and $90 \%$, respectively. Once again we observe high consistency in classification performance for training, validation, and test data. We also note that the classification performance for cluster 2 is better compared to results obtained with cluster 1 . In addition, it is also observed that misclassification of FOG event as non-FOG event is significantly higher compared to situations where non-FOG event is misclassified as FOG event.

Figure 1.9 represents the ROC curve of the feedforward network at epoch 75 for cluster 2. 


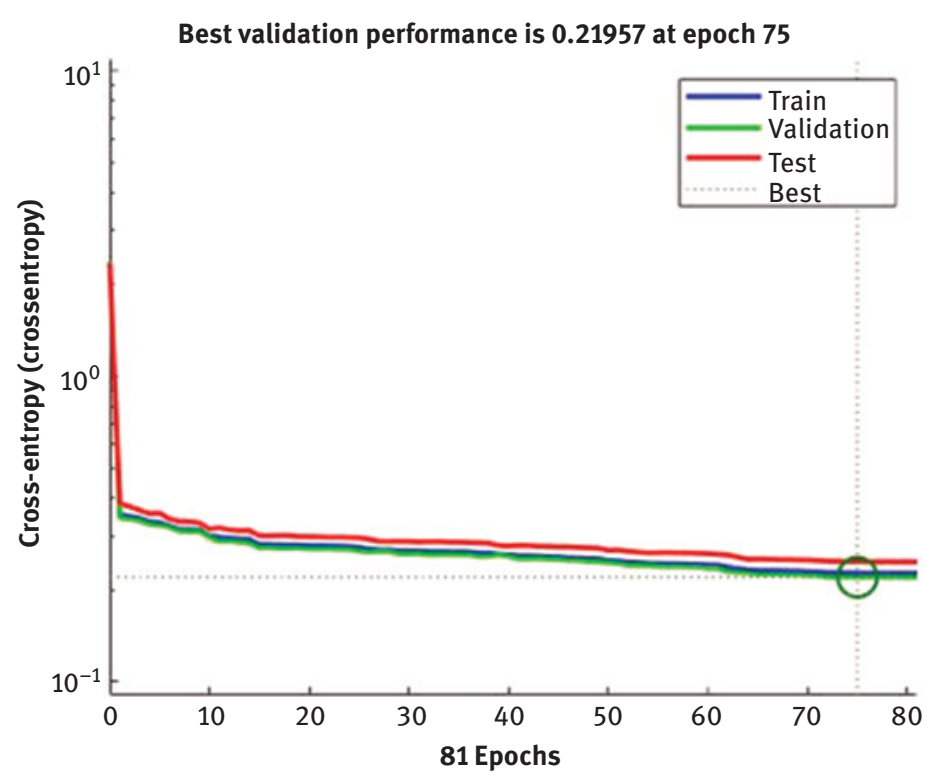

Figure 1.7: Cross-entropy versus performance using training, validation, and test data for cluster 2 .

The ROC curves based on training, validation, and test data show consistently high performance compared to the diagonal line representing 50\% accuracy.

In this chapter, we classified all patients into two clusters to enable us to develop patient-independent model. The classification model where both training and testing data belonging to the same patient is a user-dependent model, whereas the model where training is performed on a set of patients and testing is performed on another set of patients is a user-independent model [11]. When classification models are made for an individual patient, this model is likely to have better performance. However, such a model is of limited use to another patient. Developing a userindependent model is cheaper and efficient compared to a user-dependent model but accuracy of both models is less. Reasons for such lower accuracy are inconsistency in data due to different walking styles, small size of the dataset, and a limited number of patients.

\subsection{Conclusion}

A user-independent methodology for detection and prediction of FOG events using combination of clustering and neural network model is illustrated in this chapter. This chapter is divided into three stages: feature extraction, clustering, and model development/evaluation. The dataset contains data from three different sensors 

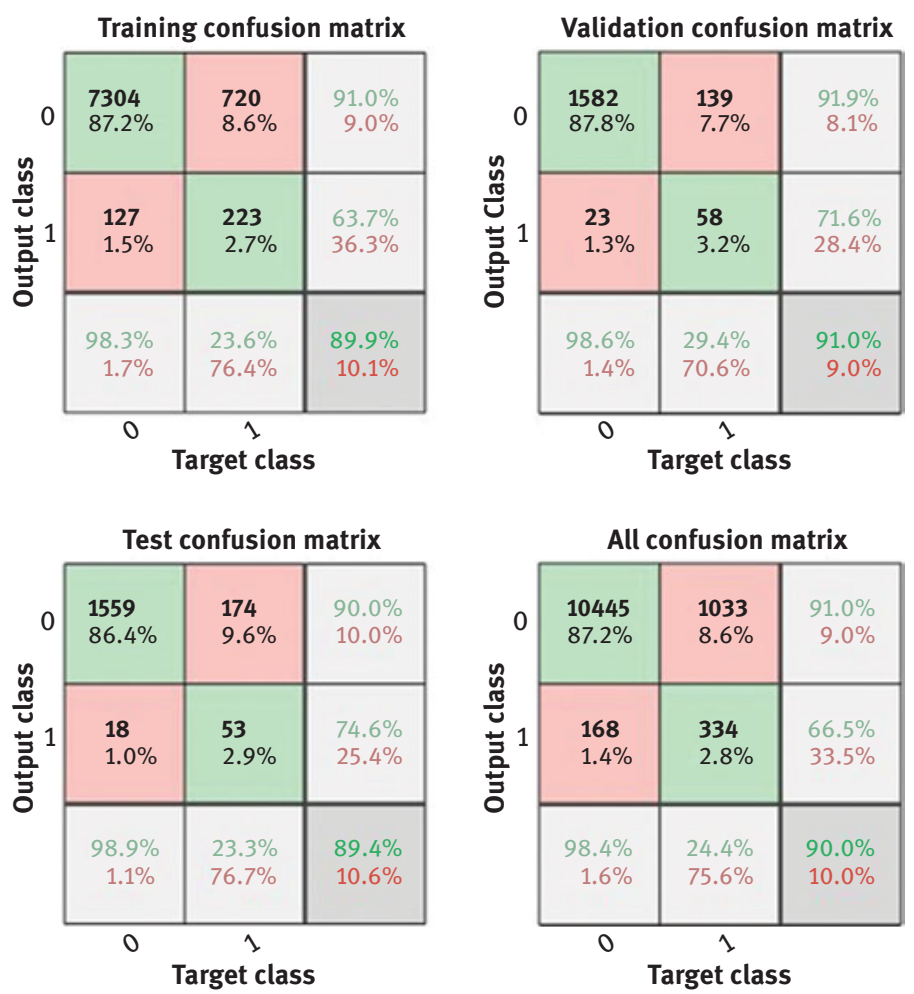

Figure 1.8: The confusion matrix for cluster 2.

that are used to derive 99 variables with the help of 11 statistical numeric summary values. An overlapping window of $50 \%$ is utilized to extract the features that were further used to group patients into two clusters using $k$-means clustering. Feedforward neural network classification model is built for each of the two clusters.

In this study, data from all sensors were utilized to perform the experiment. We used neural network-based classification model in this chapter. Further research may involve use of various other machine learning and deep learning methods to improve classification performance. In addition, this dataset used has a significantly lower number of FOG events (about 10\%) compared to no-FOG events (about 90\%). Such a severe imbalance in the two categories is likely to cause bias in results where no-FOG events are classified with a much higher accuracy compared to accuracy in classifying FOG events. This bias is observed in the classification model that was developed for cluster 1 and cluster 2. In both classification models, accuracy in correctly classifying no-FOG events is higher than $90 \%$. Whereas accuracy in correctly classifying FOG events for classification models for both cluster 1 and cluster 2 has a percentage lower than $75 \%$. This disparity in accuracy for two classes observed due to class imbalance is an interesting problem and needs further investigation. 

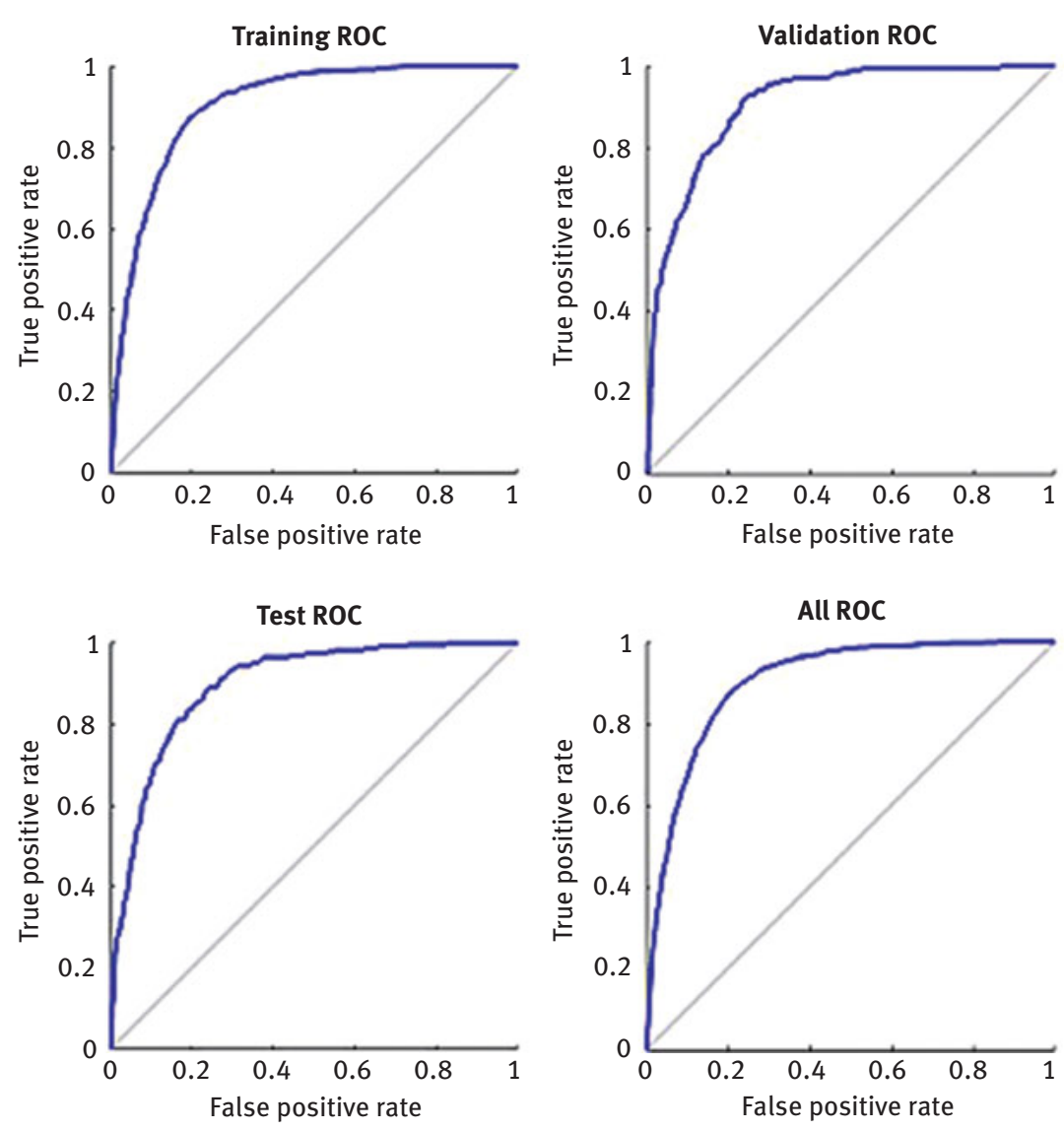

Figure 1.9: The ROC curve for a feedforward network at 75 splits for cluster 2.

\section{References}

[1] Fahn S. (2003) Description of Parkinson's disease as a clinical syndrome, Annals of the New York Academy of Sciences, 991, 1-14.

[2] Jankovic J. (2008) Parkinson's disease: clinical features and diagnosis, Journal of the Neurological Neurosurgery Psychiatry, 79(4), 368-376.

[3] Hoehn M.M. \& Yahr M.D. (1967) Parkinsonism: onset, progression, and mortality, Neurology, 17(5), 427-427.

[4] Bächlin M., Plotnik M., Roggen D., Maidan I., Hausdorff J.M., Giladi N., \& Tröster G. (2010) Wearable assistant for Parkinson's disease patients with the freezing of gait symptom, IEEE Transactions on Information Technology in Biomedicine, 14(2), 436-446.

[5] Heremans E., Nieuwboer A., \& Vercruysse S. (2013) Freezing of gait in Parkinson's disease: where are we now?, Current Neurology Neuroscience Reports, 13(6), 350. 
[6] Nutt J.G., Bloem B.R., Giladi N., Hallett M., Horak F.B., \& Nieuwboer A. (2011) Freezing of gait: Moving forward on a mysterious clinical phenomenon, Lancet Neurology, 10(8), 734-744.

[7] Schaafsma J.D., Balash Y., Gurevich T., Bartels A.L., Hausdorff J.M., \& Giladi N. (2003) Characterization of freezing of gait subtypes and the response of each to levodopa in Parkinson's disease, European Journal of Neurology, 10(4), 391-398.

[8] Okuma Y. (2014) Practical approach to freezing of gait in Parkinson's disease, The Practice of Neurology, 14(4), 222-230.

[9] Jankovic J. \& Aguilar L.G. (2008) Current approaches to the treatment of Parkinson's disease, Neuropsychiatric Disease and Treatment, 4(4), 743-757.

[10] Boelen M. (2007) The Role of rehabilitative modalities and exercise in Parkinson's disease, Disease-a-Month, 53(May), 259-264.

[11] Delval A., Snijders A.H., Weerdesteyn V., Duysens J.E., Defebvre L., Giladi N., \& Bloem B.R. (2010) Objective detection of subtle freezing of gait episodes in Parkinson's disease, Movement Disorders, 25(11), 1684-1693. 